\title{
On Prognosis of the Competitiveness of Industrial Enterprises
}

\author{
Evgeny L. Pankratovi,2,* Yu.V. Trifonov ${ }^{1}$ \\ ${ }^{1}$ Nizhny Novgorod State University \\ Nizhny Novgorod, Russia \\ ${ }^{2}$ Nizhny Novgorod State Technical University \\ Nizhny Novgorod, Russia \\ *Corresponding author's email: elp2004 [AT] mail.ru
}

\begin{abstract}
In this paper we introduce an analytical approach for prognosis of competitiveness of industrial enterprises. Based on this approach we made the considered prognosis of the competitiveness with account several factors: market capacity of manufactured products by enterprises, transport and energy costs, etc.
\end{abstract}

Keywords--- Competitiveness of industrial enterprises; prognosis of competitiveness; analytical approach for modelling

\section{INTRODUCTION}

In modern economic theory, scientific and technological progress considers as one of the relevant factors of long-term economic growth. The influence of scientific and technological progress on a particular sector of the economy appears in the creation of new products that have important competitive advantages over existing ones, or in the modification (modernization) of existing products. Often, new products based on new (innovative) technologies. However, technological superiority requires well-timed modernization of production and staff training, that is, significant financial and organizational investments. At the same time, the rejection of the transition to innovative technologies can lead to tangible losses in market positions or even to a complete cessation of the organization. Currently, one of the important examples of markets, which characterizing by the crowding out of some products by others that are more attractive from a technological point of view, is the market for information and telecommunication technologies (the market for data transmission services). For most managers of enterprises that are suppliers of products and investors, the issue of competitiveness of enterprises and their development prospects [1-10] and its prognosis [11-13] is relevant. The competitiveness of an enterprise is determined, first of all, by the quantity of manufactured products and sold, as well as the profit received. Lower costs and costs increase competitiveness. Profits and costs are affected by many factors that change over time. In this situation, it is of interest to formulate a methodology for predicting the competitiveness of enterprises. The aim of this work is the formation of such a technique, allowing to take into account the maximum possible quantity of factors at the same time.

\section{METHOD OF SOLUTION}

Let us consider several industrial enterprises producing one product with quantities $N_{i}$, where $i$ is the number of the enterprise. We describe the change in time $t$ of the quantities of products and profits of enterprises $Q_{i}(t)$ using the following system of equations 


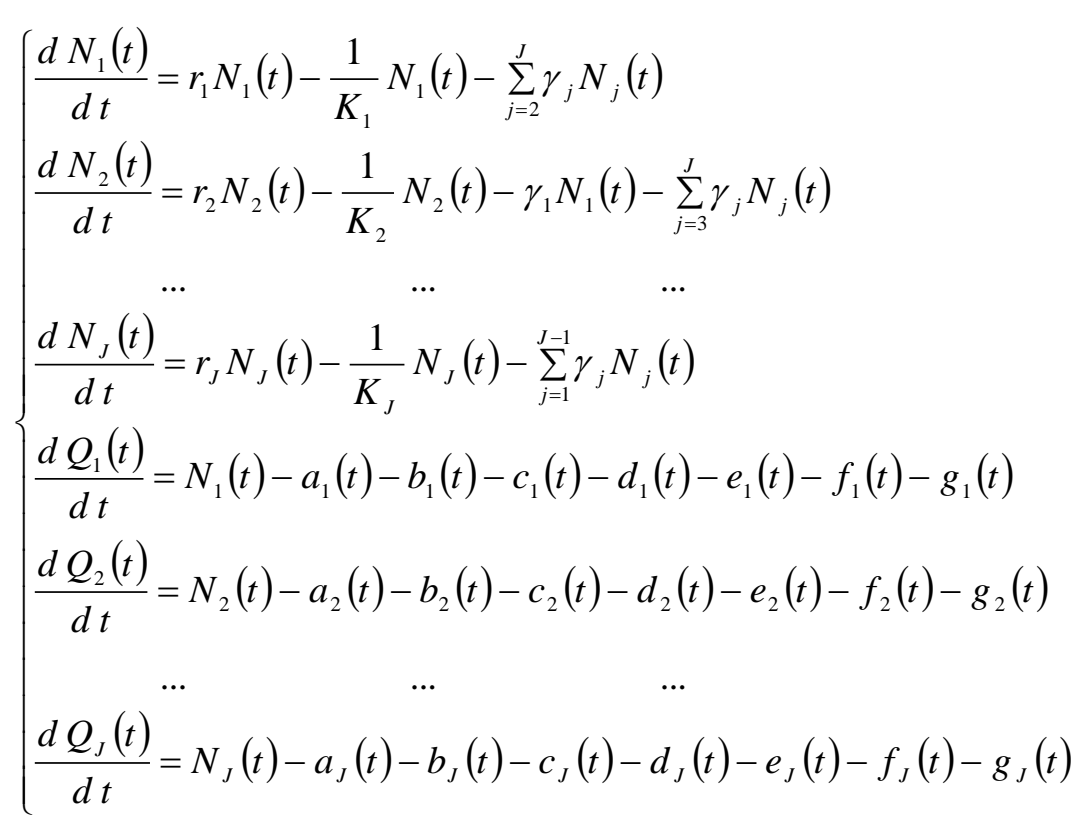

The description of the considered quantities using differential equations gives a possibility to take into account their instantaneous changes $[14,15]$ in comparison with the stationary description $[5,7,11,12]$. The following notation is introduced in the system of Eqs. (1): $K_{j}$ is the market capacity for the product $N_{j}(t) ; r_{j}$ is the parameter of growth in the quantity of products; $\gamma_{j}$ is the parameter of competition of products; $a_{j}(t)$ is the transport costs; $b_{j}(t)$ is the energy costs of production; $c_{j}(t)$ is the personnel costs (salary, training and medical expenses, but at the same time $\sum_{j=1}^{J} c_{j}(t)=c$, i.e. the number of employees is limited and they can move from one company to another); $d_{j}(t)$ is the raw materials $\left(\sum_{j=1}^{J} d_{j}(t)=d\right.$, i.e. the amount of raw materials is limited); $e_{j}(t)$ is the research costs (market research; research for the development of technology, including taking into account depreciation); $f_{j}(t)$ - tax expense; $g_{j}(t)$ is the waste disposal costs. The initial conditions for the functions $N_{j}(t), Q_{j}(t)$ are representable in the following form

$$
N_{j}(0)=\hat{N}_{j}, Q_{j}(0)=0, j=\overline{1, n} .
$$

We solve the system of Eqs. (1) by method of averaging of functional corrections [16]. In this paper as an example of competitiveness analysis we will consider the simplest case of competition between two enterprises $(j=2)$. In this case, the analysis will be most obvious. At the same time framework this procedure described below, we can generalize it to the case of a larger number of competing firms. At the first stage of solving Eqs. (1), we transform them from differential form to integral one

$$
\left\{\begin{array}{c}
N_{1}(t)=r_{1} \int_{0}^{t} N_{1}(\tau) d \tau-\frac{1}{K_{1}} \int_{0}^{t} N_{1}(\tau) d \tau-\gamma_{2} \int_{0}^{t} N_{2}(\tau) d \tau+\hat{N}_{1} t \\
N_{2}(t)=r_{2} \int_{0}^{t} N_{2}(\tau) d \tau-\frac{1}{K_{2}} \int_{0}^{t} N_{2}(\tau) d \tau-\gamma_{1} \int_{0}^{t} N_{1}(\tau) d \tau+\hat{N}_{2} t \\
Q_{1}(t)=\int_{0}^{t} N_{1}(\tau) d \tau-\int_{0}^{t} a_{1}(\tau) d \tau-\int_{0}^{t} b_{1}(\tau) d \tau-\int_{0}^{t} c_{1}(\tau) d \tau-\int_{0}^{t} d_{1}(\tau) d \tau- \\
-\int_{0}^{t} e_{1}(\tau) d \tau-\int_{0}^{t} f_{1}(\tau) d \tau-\int_{0}^{t} g_{1}(\tau) d \tau \\
Q_{2}(t)=\int_{0}^{t} N_{2}(\tau) d \tau-\int_{0}^{t} a_{2}(\tau) d \tau-\int_{0}^{t} b_{2}(\tau) d \tau-\int_{0}^{t} c_{2}(\tau) d \tau-\int_{0}^{t} d_{2}(\tau) d \tau- \\
-\int_{0}^{t} e_{2}(\tau) d \tau-\int_{0}^{t} f_{2}(\tau) d \tau-\int_{0}^{t} g_{2}(\tau) d \tau
\end{array}\right.
$$


Framework the considered approach we replace the required functions $N_{1}(t)$ and $N_{2}(t)$ in the right-hand sides of the above equations on their not yet known average values $\alpha_{11}$ and $\alpha_{21}$. Substituting these values into the equations in question allows us to obtain the first approximations of the time changes in the quantities of products $N_{11}(t)$ and $N_{21}(t)$

$$
\left\{\begin{array}{l}
N_{11}(t)=r_{1} \alpha_{11} t-\frac{1}{K_{1}} \alpha_{11} t-\gamma_{2} \alpha_{12} t+\hat{N}_{1} t \\
N_{12}(t)=r_{2} \alpha_{12} t-\frac{1}{K_{2}} \alpha_{12} t-\gamma_{1} \alpha_{11} t+\hat{N}_{2} t
\end{array}\right.
$$

We determined average values $\alpha_{i 1}$ by using following standard relations

$$
\alpha_{i 1}=\frac{1}{\Theta} \int_{0}^{\Theta} N_{i 1}(t) d t, i=1,2
$$

Substitution of relations (3) into relations (4) gives a possibility following systems of equations to determine the required average values

$$
\left\{\begin{array}{l}
\alpha_{11}=r_{1} \alpha_{11} \frac{\Theta}{2}-\frac{\alpha_{11} \Theta}{2 K_{1}}-\gamma_{2} \alpha_{12} \frac{\Theta}{2}+\hat{N}_{1} \frac{\Theta}{2} \\
\alpha_{12}=r_{2} \alpha_{12} \frac{\Theta}{2}-\frac{\alpha_{12} \Theta}{2 K_{2}}-\gamma_{1} \alpha_{11} \frac{\Theta}{2}+\hat{N}_{2} \frac{\Theta}{2}
\end{array}\right.
$$

Solution of the above system of equations could be written as

$$
\alpha_{11}=\frac{\hat{N}_{2}-\frac{\hat{N}_{1}}{\gamma_{2}}\left(\frac{2}{\Theta}-r_{2}+\frac{1}{K_{2}}\right)}{\gamma_{1}-\frac{1}{\gamma_{2}}\left(\frac{2}{\Theta}-r_{1}+\frac{1}{K_{1}}\right)\left(\frac{2}{\Theta}+\frac{1}{K_{2}}-r_{2}\right)}, \alpha_{12}=\frac{\hat{N}_{1}}{\gamma_{2}}-\frac{\left(\frac{2}{\Theta}+\frac{1}{K_{1}}-r_{1}\right)\left[\hat{N}_{2}-\frac{\hat{N}_{1}}{\gamma_{2}}\left(\frac{2}{\Theta}+\frac{1}{K_{2}}-r_{2}\right)\right]}{\gamma_{1} \gamma_{2}-\left(\frac{2}{\Theta}-r_{1}+\frac{1}{K_{1}}\right)\left(\frac{2}{\Theta}+\frac{1}{K_{2}}-r_{2}\right)} .
$$

The second-order approximations and approximations with higher orders of the required functions $N_{1}(t)$ and $N_{2}(t)$ could be determined by using the following standard replacement $N_{i 1}(t) \rightarrow \alpha_{i 1}+N_{i-11}(t)$ and $N_{i 2}(t) \rightarrow \alpha_{i 2}+N_{i-12}(t)$ in the right hands of the first and the second equations of the system $(1 a)$. The second-order approximations of the required quantities of the considered products could be written as

$$
\begin{aligned}
N_{21}(t)=\alpha_{21} t\left(r_{1}-\frac{1}{K_{1}}\right) & +\alpha_{22} t\left(r_{1} r_{2} \frac{t}{2}-\gamma_{2}\right)+\alpha_{11} \frac{t^{2}}{2}\left(\gamma_{2} \gamma_{1}-2 \frac{r_{1}}{K_{1}}-\frac{1}{K_{1}^{2}}\right)-\frac{\hat{N}_{1} t^{2}}{2 K_{1}}-\gamma_{2} \hat{N}_{2} \frac{t^{2}}{2}+ \\
& +\alpha_{12} \gamma_{2} \frac{t^{2}}{2}\left(\frac{K_{1}+K_{2}}{K_{1} K_{2}}-r_{1}-r_{2}\right)+r_{1} \hat{N}_{1} \frac{t^{2}}{2}+\hat{N}_{1} t \\
N_{22}(t)=\alpha_{22} t\left(r_{2}-\frac{1}{K_{2}}\right)+ & \alpha_{11} \frac{t^{2}}{2}\left(\gamma_{1} \frac{K_{1}+K_{2}}{K_{1} K_{2}}-r_{2} \gamma_{1}-r_{1} \gamma_{1}\right)+\alpha_{12} \frac{t^{2}}{2}\left(r_{2}^{2}-2 \frac{r_{2}}{K_{2}}+\frac{1}{K_{2}^{2}}+\gamma_{1} \gamma_{2}\right)+(7) \\
& +r_{2} \hat{N}_{2} \frac{t^{2}}{2}-\frac{\hat{N}_{2} t^{2}}{2 K_{2}}-\gamma_{1} \hat{N}_{1} \frac{t^{2}}{2}+\hat{N}_{2} t-\alpha_{21} \gamma_{1} t .
\end{aligned}
$$

We calculate not yet known average values $\alpha_{i j}$ (order of approximation is $i \geq 2$ ) by using the following standard relations

$$
\alpha_{i j}=\frac{1}{\Theta} \int_{0}^{\Theta}\left[N_{i j}(t)-N_{i j-1}(t)\right] d t ; i \geq 2 ; j=1,2 .
$$


Substitution of relations (7) into relation (8) gives a possibility to obtain system of equations to determine required average values

$$
\begin{array}{r}
\alpha_{21}=\alpha_{21} \frac{\Theta}{2}\left(r_{1}-\frac{1}{K_{1}}\right)+\alpha_{22} \frac{\Theta}{2}\left(r_{1} r_{2} \frac{\Theta}{3}-\gamma_{2}\right)+\alpha_{11} \frac{\Theta}{2}\left(\gamma_{2} \gamma_{1} \frac{\Theta}{3}-2 \frac{r_{1} \Theta}{3 K_{1}}-\frac{\Theta}{3 K_{1}^{2}}-r_{1}+\frac{1}{K_{1}}\right)-\hat{N}_{1} \frac{\Theta}{2}+ \\
+\alpha_{12} \gamma_{2} \frac{\Theta}{2}\left(\frac{\Theta}{3} \frac{K_{1}+K_{2}}{K_{1} K_{2}}-r_{1} \frac{\Theta}{3}-r_{2} \frac{\Theta}{3}+1\right)+\hat{N}_{1} \frac{\Theta}{2}+r_{1} \hat{N}_{1} \frac{\Theta^{2}}{6}-\frac{\hat{N}_{1} \Theta^{2}}{6 K_{1}}-\gamma_{2} \hat{N}_{2} \frac{\Theta^{2}}{6} \\
\alpha_{22}=-\alpha_{21} \gamma_{1} \frac{\Theta}{2}+\alpha_{22} \frac{\Theta}{2}\left(r_{2}-\frac{1}{K_{2}}\right)+\alpha_{11} \gamma_{1} \frac{\Theta}{2}\left(\frac{K_{1}+K_{2}}{K_{1} K_{2}} \frac{\Theta}{3}-r_{2} \frac{\Theta}{3}-r_{1} \frac{\Theta}{3}+1\right)+r_{2} \hat{N}_{2} \frac{\Theta^{2}}{6}-\hat{N}_{2} \frac{\Theta}{2}-(9) \\
-\frac{\hat{N}_{2} \Theta^{2}}{6 K_{2}}+\alpha_{12} \frac{\Theta}{2}\left(r_{2}^{2} \frac{\Theta}{3}-2 \frac{r_{2} \Theta}{3 K_{2}}+\frac{\Theta}{3 K_{2}^{2}}+\gamma_{1} \gamma_{2} \frac{\Theta}{3}-r_{2}+\frac{1}{K_{2}}\right)+\hat{N}_{2} \frac{\Theta}{2}+\gamma_{1} \hat{N}_{1} \frac{\Theta}{6} .
\end{array}
$$

Solution of system of Eqs. (9) could be written as

$$
\alpha_{21}=\frac{b_{1} a_{22}-a_{12} b_{2}}{a_{11} a_{22}-a_{12} a_{21}}, \alpha_{22}=\frac{a_{11} b_{2}-b_{1} a_{21}}{a_{11} a_{22}-a_{12} a_{21}},
$$

where $a_{11}=1+\frac{\Theta}{2 K_{1}}-r_{1} \frac{\Theta}{2}, a_{11}=\frac{\Theta}{2}\left(\gamma_{2}-r_{1} r_{2} \frac{\Theta}{3}\right), a_{21}=\gamma_{1} \frac{\Theta}{2}, a_{22}=1+\frac{\Theta}{2 K_{2}}-r_{2} \frac{\Theta}{2}, b_{1}=r_{1} \hat{N}_{1} \frac{\Theta^{2}}{6}-$

$-\frac{\hat{N}_{1} \Theta^{2}}{6 K_{1}}+\alpha_{11} \frac{\Theta}{2}\left(\gamma_{2} \gamma_{1} \frac{\Theta}{3}-2 \frac{r_{1} \Theta}{3 K_{1}}-\frac{\Theta}{3 K_{1}^{2}}-r_{1}+\frac{1}{K_{1}}\right)+\alpha_{12} \gamma_{2} \frac{\Theta}{2}\left(\frac{\Theta}{3} \frac{K_{1}+K_{2}}{K_{1} K_{2}}-r_{1} \frac{\Theta}{3}-r_{2} \frac{\Theta}{3}+1\right)+$

$+\hat{N}_{1} \frac{\Theta}{2}-\gamma_{2} \hat{N}_{2} \frac{\Theta}{6}-\hat{N}_{1} \frac{\Theta}{2}, b_{2}=\alpha_{11} \gamma_{1} \frac{\Theta}{2}\left(\frac{K_{1}+K_{2}}{K_{1} K_{2}} \frac{\Theta}{3}-r_{2} \frac{\Theta}{3}-r_{1} \frac{\Theta}{3}+1\right)+\hat{N}_{2} \frac{\Theta}{2}+\gamma_{1} \hat{N}_{1} \frac{\Theta^{2}}{6}+$

$+\alpha_{12} \frac{\Theta}{2}\left(r_{2}^{2} \frac{\Theta}{3}-2 \frac{r_{2} \Theta}{3 K_{2}}+\frac{\Theta}{3 K_{2}^{2}}+\gamma_{1} \gamma_{2} \frac{\Theta}{3}-r_{2}+\frac{1}{K_{2}}\right)+r_{2} \hat{N}_{2} \frac{\Theta^{2}}{6}-\frac{\hat{N}_{2} \Theta^{2}}{6 K_{2}}-\hat{N}_{2} \frac{\Theta}{2}$.

In this paper the required quantities of products were determined by using the second-order approximation framework the method of averaging functional corrections. The approximation is usually sufficient to obtain qualitative conclusions and obtain some quantitative results.

Solution to the second pair of equations of the system (1) was determined by the integration of the left and right sides. As a result of this integration is

$$
\left\{\begin{array}{r}
Q_{1}(t)=\int_{0}^{t} N_{1}(\tau) d \tau-\int_{0}^{t} a_{1}(\tau) d \tau-\int_{0}^{t} b_{1}(\tau) d \tau-\int_{0}^{t} c_{1}(\tau) d \tau-\int_{0}^{t} d_{1}(\tau) d \tau- \\
\quad-\int_{0}^{t} e_{1}(\tau) d \tau-\int_{0}^{t} f_{1}(\tau) d \tau-\int_{0}^{t} g_{1}(\tau) d \tau \\
Q_{2}(t)=\int_{0}^{t} N_{2}(\tau) d \tau-\int_{0}^{t} a_{2}(\tau) d \tau-\int_{0}^{t} b_{2}(\tau) d \tau-\int_{0}^{t} c_{2}(\tau) d \tau-\int_{0}^{t} d_{2}(\tau) d \tau- \\
-\int_{0}^{t} e_{2}(\tau) d \tau-\int_{0}^{t} f_{2}(\tau) d \tau-\int_{0}^{t} g_{2}(\tau) d \tau
\end{array}\right.
$$

\section{DISCUSSION}

In this section we analyze influence of various parameters on change in time of the considered quantities of manufactured products and profits, which were received from their sale. Figs. 1-3 show typical qualitative dependences 
of the quantity of considered products on various parameters (on time, market capacity, growth parameter of the quantity of goods). These dependences of considered quantity of products on the competition parameter of two products are similar to its dependencies on the growth parameter of the quantity of products. Profit will change faster over time than the amount of products produced. Its dependence on the market capacity, growth parameter of the quantity of products and the competition parameter of two products are similar to the dependencies shown in Figs. 1-3. Analysis of the dependence of profit on the parameters shows that there are combinations of parameters for which $(i)$ the profit of the enterprise always exists and increases; (ii) the profit of the enterprise decreases and the production of goods gradually loses profitability; (iii) production is temporarily unprofitable and gradually becomes profitable with the development of the enterprise. During manufacturing of product some natural economical special cases: $(i)$ with a well-chosen strategy, there will be a stable growth in the manufacturing of product; (ii) with a less well-chosen strategy the manufacturing of product ceases due to market saturation or because of the too high cost of product; (iii) there may also be a delay in the manufacturing of product due to the necessity in preparation of technological process.

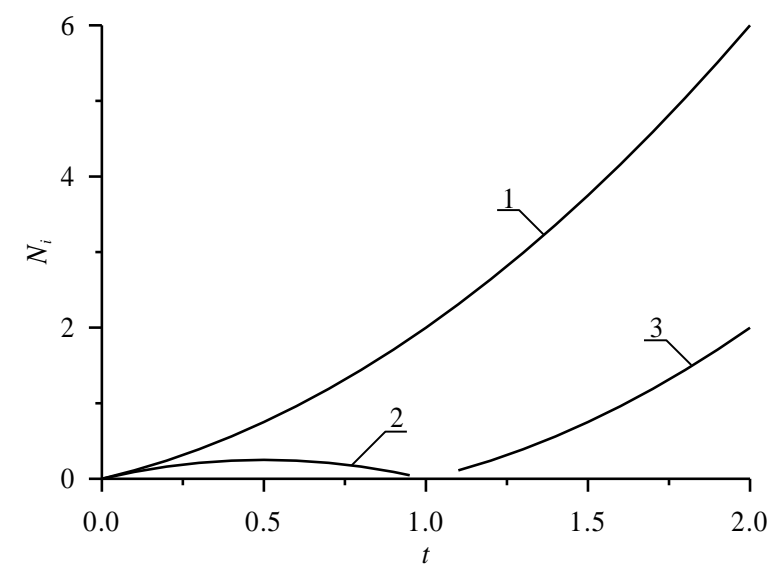

Fig. 1. Qualitative dependencies of the quantity of manufactured products on time

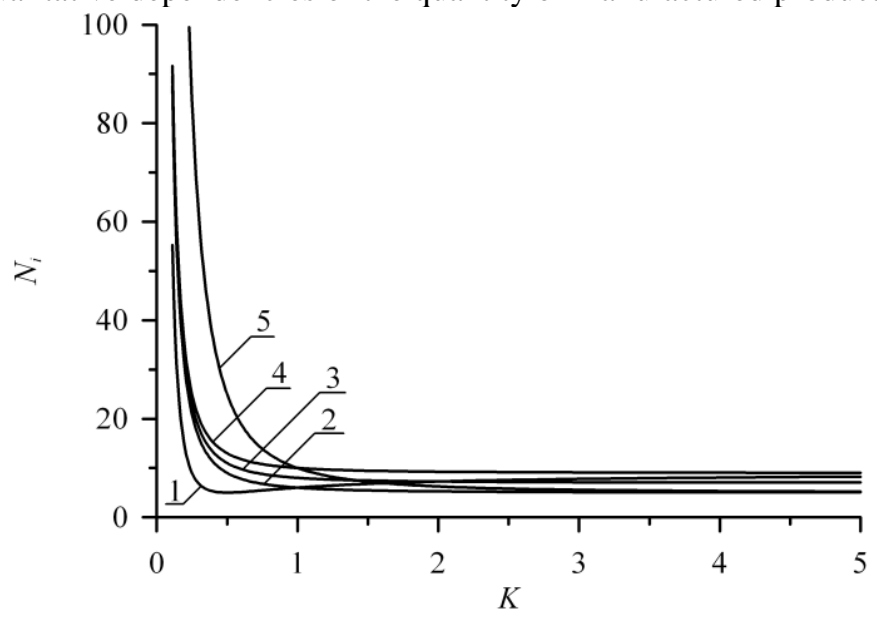

Fig. 2. Qualitative dependencies of the quantity of manufactured products on market capacity

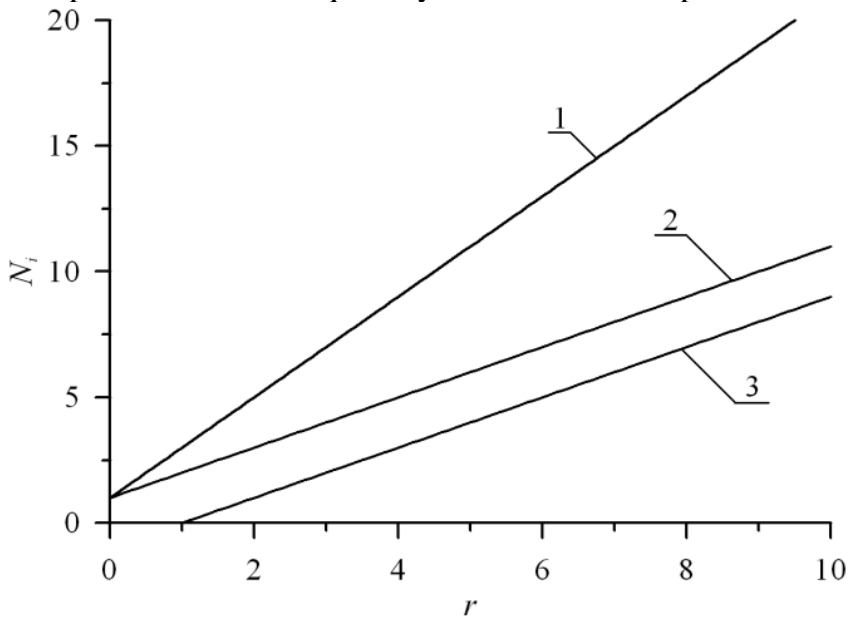


Fig. 3. Qualitative dependencies of the quantity of manufactured products on growth parameter of these products

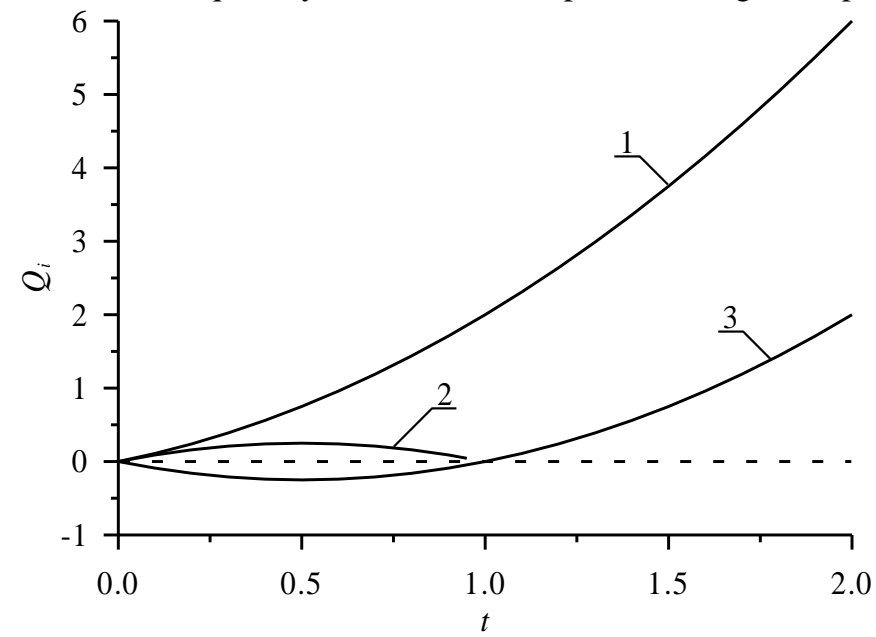

Fig. 4. Qualitative dependencies of the quantity of profit on time

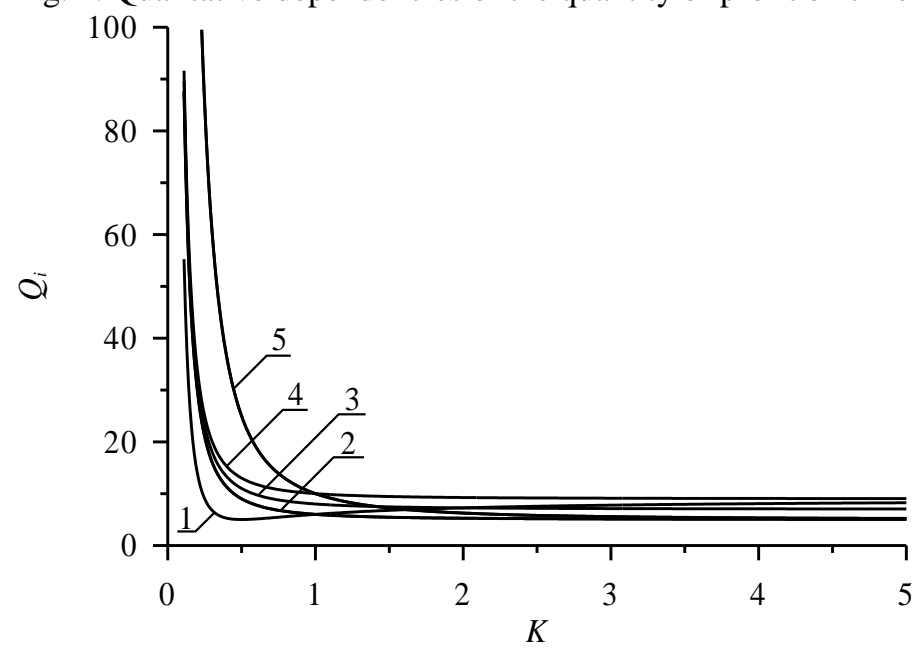

Fig. 5. Qualitative dependencies of the quantity of profit products on market capacity

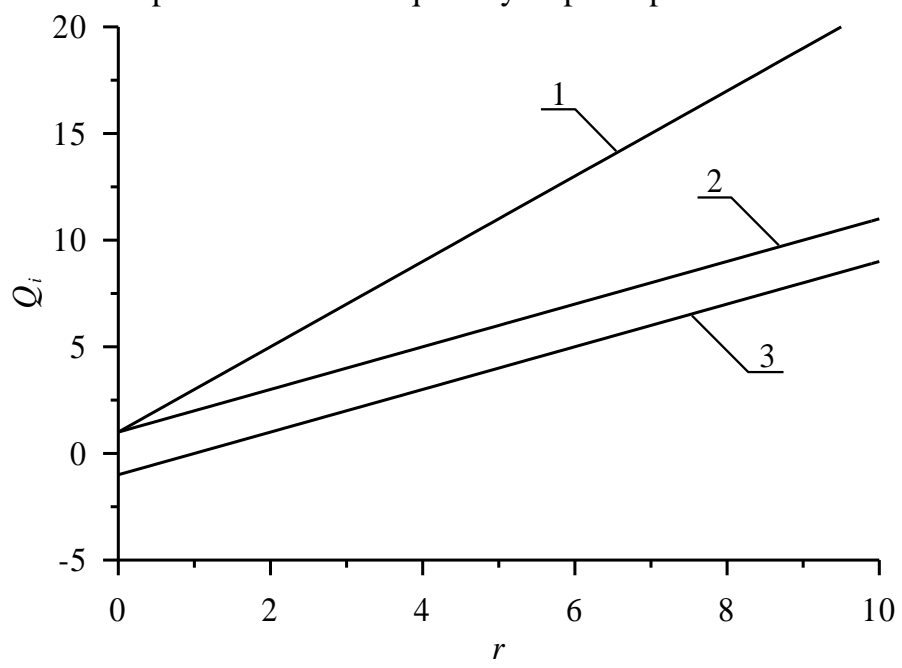

Fig. 6. Qualitative dependencies of the quantity of profit on growth parameter of these products

\section{CONCLUSION}

In this paper, we introduce a model for prognosis of competitiveness of industrial enterprises with account instantaneous changes of required values and parameters their parameters. We also introduce an analytical approach to solve equations, which were used in our model, taking into account possible changes of its parameters on time (transport costs, personnel costs, taxes, etc.). The analysis of the introduced model has been done. As a result of the analysis we 
obtain following limiting cases, which are native from economical point of view: (i) if the product manufacturing strategy is successfully chosen, the company will have a stable profit; with less well-chosen strategies, the enterprise (ii) will have temporary losses related to the need to organize the manufacturing of products, or (iii) the manufacturing of products will stop due to market saturation or too high cost of the products manufactured. In the end, the results of the performed analysis allow the company to determine the rational way of further functioning and the choice of the most appropriate way of strategic development.

\section{REFERENCES}

[1] Mandal S. "The influence of organizational culture on healthcare supply chain resilience: moderating role of technology orientation", Journal of business and industrial marketing, vol. 32, № 8, pp. 1021-1037, 2017.

[2] Phooma J., Tongliemnak K., Songchalearnkul Pr., Chanin O., and Poomusub, " The behavior of local food consumption in nakhon sri thammarat province by applying of folk wisdom", International journal of innovation management, vol. 7, № 4, pp. 150-167, 2016.

[3] Ple L., Lecocq X., " Customers as creative resources: their influence on firm freedom ", Journal of Business Strategy, vol. 36, № 4, pp. 11-22 (2015).

[4] Lynette J.R., Iain A.D. "Where's the strategic intent in key account relationships?", Journal of business and industrial marketing, vol. 28, № 2, pp. 111-124, 2013.

[5] Das J., Nath D.C. "Burr distribution as an actuarial risk model and the computation of some of its actuarial quantities related to the probability of ruin", Journal of mathematical finance, vol. 6, № 1, pp. 213-231, 2016.

[6] Jeong S.-Y. "A study on the decision making of new construction technology information services development project using real option model", International Journal of Innovation Management, vol. 8, № 1, pp. 71-76, 2017.

[7] Wu Ch.W., Hung W.Zh. "Real national income average growth rate: a novel economic growth and social fair evaluation index", Economics research international, Vol. 2010, Article ID 678927, 2010.

[8] Yaghoubi R., Yaghoubi M., Locke St., Gibb J. "Mergers and acquisitions: a review (part 2)", Studies in economics and finance, vol. 33, № 3, pp. 437-464, 2016.

[9] J. Prat. Dynamic contracts and learning by doing, "Mathematics and financial economics", vol. 9, № 3, pp. 169-193, 2015.

[10] Trifonov Yu.V., Maslova T.E., Trifonova E.Yu. "Strategic analysis and integrated assessment of the state of economic systems", Basic research, № 5, pp. 143-147, 2019.

[11] Trachuk A.V., Linder N.V. "Innovation activity of industrial companies: measuring and evaluating effectiveness", Strategic decisions and risk management, vol.10, № 2, pp. 108-121, 2019.

[12] Abdikeev N.M., Bogachev Yu.S., Losev A.A., Tolkachev S.A. "Multifunctional model and competitiveness assessment of socio-economic systems", Strategic decisions and risk management, vol.10, № 2, pp. 169-193, 2019.

[13] Trifonov Yu.V., Skibin A.G. " Strategic analysis and level assessment competitiveness of oil companies", Bulletin of Nizhny Novgorod state university, № 2, pp. 31-39, 2018.

[14] Pankratov E.L. " An analytical approach to the analysis of industrial enterprise activity", Journal of coupled systems and multiscale dynamics, vol. 6, № 2, pp. 154-157, 2018.

[15] Pankratov E.L. "Model for prognosis of economic growth: accounting of influence of the environment", Global economy journal, vol. 19, № 1, pp. 1950007, 2019.

[16] Sokolov Yu.D. "About the definition of dynamic forces in the mine lifting", Applied Mechanics, vol. 1, № 1, pp. $23-$ $35,1955$. 